Para enlazar con este artículo / To link to this article:

http://dx.doi.org/10.14198/fem.2018.31.06

Para citar este artículo / To cite this article:

Martínez Sanz, Alicia. «¿Por qué las mujeres tienen relaciones sexuales con sus parejas cuando realmente no lo desean?». En Feminismo/s, 31 (junio 2018): 127-141. Dosier monográfico: Sexo y bienestar. Mujeres y diversidad, coords. Carmen Mañas Viejo y Alicia Martínez Sanz, DOI: 10.14198/fem.2018.31.06

\title{
¿POR QUÉ LAS MUJERES TIENEN RELACIONES SEXUALES CON SUS PAREJAS CUANDO REALMENTE NO LO DESEAN?
}

\section{WHY DO WOMEN HAVE SEXUAL RELATIONSHIPS WITH THEIR PARTNERS WHEN THEY REALLY DO NOT WANT IT?}

\author{
Alicia MARTÍNEZ SANZ \\ Departamento de Psicología Evolutiva y Didáctica. Facultad de Educación. \\ Universidad de Alicante \\ orcid.org/0000-0001-7212-5775
}

\section{Resumen}

Muchas mujeres mantienen relaciones sexuales con sus parejas realizando conductas que no les agradan y con una frecuencia muy por encima de lo que realmente desean; sienten la obligación de hacerlo y creen que es algo autoimpuesto. Sin embargo, en realidad viven en una continua situación de violencia sexual, aunque no lo perciben como tal. Por ello, en este ensayo científico ponemos al descubierto esta realidad y exponemos algunas de las razones que influyen en las mujeres para que, una y otra vez, mantengan relaciones sexuales con sus parejas bajo coacción, sin ser conscientes de los mecanismos y las consecuencias que subyacen a tales hechos. Entre estos motivos destacamos las creencias que acompañan a los roles de género, en concreto al rol sexual femenino como, por ejemplo, la creencia en el «débito conyugal».

Palabras clave: violencia sexual, falta de percepción, violencia de género, sexualidad de las mujeres, rol sexual, débito conyugal. 


\begin{abstract}
Many women have sexual relations with their partners but doing behaviors that they don't like and with a frequency that they really don't want; they feel the obligation to do it and believe that it is self-imposed. Actually, they live in a continuous sexual violence situation, although they don't perceive it as such. Therefore, in this scientific essay, we expose this reality and expose some of the reasons that influence women to have sex with their partners under duress, again and again, without being aware of the mechanisms and consequences that underlie these events. Among these reasons, we highlight the beliefs that accompany gender roles, specifically the female sexual role, such as, for example, the «conjugal debit» belief.
\end{abstract}

Keywords: sexual violence, lack of perception, gender violence, women sexuality, sexual role, conjugal debit.

\title{
1. INTRODUCCIÓN
}

Cuando se habla de la sexualidad de las mujeres, se hace referencia a un derecho fundamental y a un estado de bienestar físico, emocional, mental y social que requiere un acercamiento positivo y respetuoso hacia la sexualidad y las relaciones sexuales, así como la posibilidad de obtener placer y experiencias sexuales seguras, libres de coerción, discriminación y violencia (OMS, Defining sexual health 4). Sin embargo, cuando se estudia la sexualidad de las mujeres, aparece una mácula que, lamentablemente, se vincula a la misma: la violencia sexual.

La violencia sexual es un tipo de violencia de género que comprende una gran diversidad de actos, como las relaciones sexuales bajo coacción en el matrimonio y en las citas, las violaciones por parte de extraños, las violaciones sistemáticas durante los conflictos armados, el acoso sexual, los abusos sexuales de menores, los matrimonios precoces y los actos violentos contra la integridad sexual de las mujeres, como la mutilación genital y las inspecciones obligatorias de la virginidad (OPS 21).

Entre las diferentes formas de violencia sexual, la violencia sobre las mujeres en la relación de pareja, que es la que nos ocupa, es entendida como el tipo de violencia que se ejerce contra las mujeres y que comprende cualquier intimidad sexual forzada por parte de la pareja, ya sea con amenazas, intimidación, coacción, o por llevarse a cabo en estado de inconsciencia o 
indefensión de la mujer (Labrador, Rincón, de Luis y Fernández-Velasco 26) y es considerada una de las formas de violencia de género más extendidas en el mundo (Sanmartín, Iborra, García y Martínez). Los datos estadísticos de las investigaciones que se presentan a continuación evidencian esta realidad.

El informe multipaís de la OMS sobre salud de la mujer y violencia doméstica contra la mujer del año 2005, indica que la tasa de prevalencia de la violencia sexual infligida por la pareja oscila entre un $6 \%$ y un $59 \%$, siendo Japón el país que registra el nivel más bajo de violencia sexual (6\%) y Etiopía el más elevado (59\%). En el informe mundial sobre la violencia y la salud de la OMS (Jewkes, Sen y García-Moreno), revisa diferentes investigaciones realizadas entre 1989 y 2000 en diferentes países del mundo y obseva que los porcentajes de violencia sexual dentro de la relación de pareja oscilan entre el 5,9\% en Finlandia y el 46,7\% en Perú.

Un estudio llevado a cabo en Estados Unidos a través de encuestas telefónicas, con una muestra de 1.108 mujeres, revela que el 34\% había sufrido algún tipo de coerción sexual por su marido o por su pareja a lo largo de su vida (Basile).

Otro trabajo llevado a cabo con pacientes en dos centros de salud de Columbia S. C., descubre que el 23,1\% de las mujeres habían sufrido violencia sexual por sus parejas (Coker, Smith, Bethea, McKeown y King).

Una investigación realizada por el Instituto Nacional de las Mujeres de México, observa que el 7,8\% de las mujeres refieren haber vivido alguna forma de violencia sexual por sus parejas en el último año. De éstas, el 93,9\% afirma que sus parejas les habían exigido tener relaciones sexuales aunque ellas no quisieran y, en el 28,5\% de los casos, las parejas utilizaron la fuerza física para obligarlas. En otro estudio también mejicano, realizado en un centro de salud, concluye que el $20 \%$ de las mujeres había experimentado algún tipo de violencia sexual dentro de la relación de pareja y el 13\% de las mujeres entrevistadas refiere haber sufrido al menos una forma de violencia sexual por parte de la pareja en el último año (Ramos-Lira et al.).

En nuestro país, según las estadísticas publicadas por el Instituto de la Mujer ${ }^{1}$ sobre delitos conocidos contra la libertad e indemnidad sexual

1. Estudio elaborado a partir de los datos facilitados por el Ministerio del Interior y en el País Vasco, Girona y Lleida solo se recogen las denuncias tramitadas por los Cuerpos y Fuerzas de seguridad del Estado. 
cometidos en el ámbito familiar, durante el año 2007, el 51,74\% de las mujeres víctimas manifestó que el perpetrador era su cónyuge o un análogo. Fontanil y sus colaboradoras/es hallan que el 2,4\% de las mujeres que participaron en su investigación, llevada a cabo en población general, había recibido ataques contra su intimidad sexual a lo largo de su relación de pareja. En otra investigación realizada en centros rurales y urbanos de atención primaria, con 385 mujeres que tuvieran o hubieran tenido pareja (Cano et al.), encuentran que el $1,9 \%$ de las mujeres había vivido abuso sexual por su pareja actual y el 6,2\% en el pasado. Por último, señalar un estudio llevado a cabo en población universitaria, el cual advierte que el 30,4\% de las mujeres encuestadas había mantenido relaciones sexuales sin desearlo (Santos-Iglesias y Sierra).

El porcentaje de mujeres se eleva exponencialmente cuando se trata de población de mujeres que han sufrido otras formas de violencia en la pareja (maltrato físico y/o psicológico). En un estudio de caso realizado en 2013, contempla una prevalencia de violencia sexual del 71,43\% (Martínez Sanz, Mañas Viejo y Montesinos Sánchez).

Sin embargo, tal y como ha quedado justificado en la tesis doctoral realizada por Martínez Sanz, en la cual se basa este artículo, la violencia sexual en la pareja es invisible, porque no es percibida, ni siquiera por las mujeres que la viven cada día y consideran que el comportamiento de sus parejas cuando las presionan y las coaccionan para tener relaciones sexuales, es «normal»y que ellas «deben» acceder (343).

Por todo ello, en el desarrollo de este ensayo científico, profundizaremos en las causas que subyacen cuando las mujeres acceden a tener relaciones sexuales de forma y con una frecuencia que no desean, cuando quienes las obligan son sus propias parejas, poniendo el punto de mira en las creencias sexuales que asientan los roles de género. Así mismo, veremos como la invisibilidad que acompaña a este tipo de violencia está vinculada a la escasez de investigación y de herramientas para identificarla y explorarla en profundidad. 


\section{DESARROLLO}

A lo largo de la experiencia adquirida durante años trabajando en centros públicos especializados en la atención a la mujer ${ }^{2}$, hemos podido comprobar cómo muchas mujeres que niegan haber sido obligadas a tener relaciones sexuales en el contexto de la pareja, afirman con rotundidad que mantienen relaciones sexuales de manera habitual sin desearlo, porque sienten la imposición y por evitar las consecuencias negativas de negarse a ello, ¿no es esto sino una perversa contradicción? Es decir, no perciben que se les obliga, pero se sienten obligadas, además se creen inadecuadas y poco valiosas si no acceden y/o temen la respuesta de sus parejas si se oponen a tal mandato, por lo que acaban cediendo.

Saltijeral, Ramos y Caballero (15) afirman que la violencia sexual dentro de la relación de pareja es sumamente difícil de reconocer en nuestra sociedad, debido a que, con frecuencia, las mujeres no tienen la posibilidad de ejercer su derecho a decidir cuándo y cómo tener relaciones sexuales, por lo que, si los hombres deciden tener relaciones sexuales, las mujeres deben acceder, incluso cuando antes las han golpeado. Así mismo, Asensio refiere que «igual que en otros aspectos, en materia de sexo, la mujer, puesto que era una pertenencia del hombre, no ha podido ser dueña de sí misma, ni de su cuerpo, es decir, no ha sido libre, ni ha podido tener iniciativa» (14).

Entre los motivos que pueden influir en las mujeres para que tengan relaciones sexuales con sus parejas bajo la coacción y sin libertad para elegir plenamente, encontramos las creencias que existen sobre los roles de género. Las expectativas que se ponen sobre las mujeres como exigencias de su rol femenino, son determinantes a la hora de decidir tener o no relaciones sexuales, así como en la percepción de la violencia sexual. Una de las creencias que pensamos que más pueden influir en este aspecto es la creencia del «débito conyugal» que, aunque parezca algo del pasado, forma parte de la herencia de la cultura judeocristiana a la que pertenecemos. El débito conyugal supone una prescripción que obliga a las mujeres a mantener relaciones sexuales

2. Estos servicios son: un servicio público autonómico que ofrece atención psicológica, jurídica y social en la Comunidad Valenciana especializado en víctimas de violencia de género y otro servicio municipal, situado en una localidad de Alicante, especializado en atención psicológica y jurídica a las mujeres.

Feminismo/s 31, junio 2018, pp. 127-141 
con su marido (López Mondéjar 126) o con la pareja, por lo que las mujeres «deben» mantener relaciones sexuales aunque no lo deseen, y si se niegan, sus maridos pueden forzarlas a complacerlos porque es su deber. Labrador et al. afirman que, en consecuencia, «muchas mujeres piensan que no pueden negarse a realizar este tipo de conductas con su pareja, aunque no lo deseen, e incluso aunque sean violentamente exigidas, por lo que es poco frecuente que las mujeres consideren que han padecido violencia sexual por su pareja» (26). En este sentido, Durán, en su tesis doctoral, afirma que las mujeres se sienten culpables si rechazan satisfacer las necesidades sexuales de sus parejas, pudiendo ser su comportamiento considerado como trasgresor del rol femenino. Esta culpabilidad tiene su origen en el sexismo benévolo ${ }^{3}$, aunque también el sexismo hostil ${ }^{4}$, de los hombres, que influye en las mujeres. Es más, de su investigación sugieren que «los mayores niveles de culpabilidad atribuidos a la víctima cuando el perpetrador era caracterizado con actitudes sexistas benévolas, se debían precisamente a las características definitorias del sexismo benévolo, y no a un mero efecto de agradabilidad suscitado por el tinte afectivo del agresor benévolo» (239). En esta tesis, también se afirma que «la adhesión a la ideología sexista benévola por parte de los participantes predijo la no identificación de un acto sexual forzado como violación, porque percibían mayores derechos maritales en el marido y mayores deberes maritales en ella» (240). Así mismo, Durán sostiene que el sexismo benévolo debilita las reacciones de las mujeres ante actos sexuales abusivos por parte de sus parejas íntimas y afecta a la decisión de no abandonar una relación abusiva, y a los hombres les activaba la tendencia a involucrarse en agresiones sexuales contra sus parejas. Es decir, podemos entender que las creencias asociadas a los roles de género que derivan del sexismo, como el débito conyugal, influye en las mujeres para que se sientan obligadas a mantener relaciones con sus parejas, a sentirse culpables si no lo hacen; además, disminuye la percepción de la violencia sexual que pueden estar viviendo y aumenta las dificultades

3. Entienden el 'sexismo benévolo' como aquellas ideas y comportamientos aparentemente «positivos» que tienen los hombres hacia las mujeres y que hacen alusión a la necesidad de cuidar y sacrificarse por ellas.

4. Entienden el 'sexismo hostil' (tal y como lo define Cameron, 1977), como actitud de prejuicio o conducta discriminatoria basada en la supuesta superioridad o diferencia de las mujeres como grupo.

Feminismo/s 31, junio 2018, pp. 127-141 
para reaccionar y defender sus derechos sexuales. Estas creencias también afectan a los hombres, quienes además de no percibir la violencia sexual, la justifican apelando a su necesidad.

Cuando se pregunta a las mujeres si consideran que tienen derecho a negarse a tener relaciones sexuales con sus maridos, muy pocas se sienten cómodas o identifican una excusa para defender su preferencia, es decir, no querer tenerlas, como si esto no fuera suficiente. Piensan que está disculpado negarse a tener relaciones sexuales en determinadas situaciones como, por ejemplo, si están enfermas, si su marido está borracho o si las maltrata (OMS estudio multipaís). En esta misma investigación encuentran que, en algunos entornos provinciales de Bangladesh, Perú y República Unida de Tanzania, así como en Etiopía y Samoa, entre el 10\% y el 20\% de las mujeres llega a manifestar que no tienen derecho a negarse a tener relaciones sexuales en ninguna de las circunstancias descritas.

En un estudio similar realizado por Basile en Estados Unidos sobre la violación en las mujeres y otros tipos de coerción sexual dentro de la relación de pareja, se observa que el $61 \%$ de las mujeres que viven situaciones en las que no quieren mantener relaciones sexuales con sus parejas, acceden porque piensan que es su deber. Es decir, si las mujeres han interiorizado que satisfacer las demandas sexuales de sus parejas es su obligación, para negarse a ello necesitan una «justificación de peso» y no entra dentro de esta categoría su preferencia o deseo.

A la interiorización de los roles sexuales de las mujeres definidos por la subordinación a los deseos de los hombres, se unen otros factores como las falsas creencias relacionadas con el rol masculino. Un claro ejemplo es la supuesta «necesidad imperiosa de los hombres de tener relaciones sexuales en pareja», que también justifica la exigencia sexual de los hombres hacia las mujeres. Es decir, se entiende que es una necesidad para él y una obligación para ella y, de lo contrario, él la forzará o estará justificado que se marche con otra mujer para tener relaciones sexuales (Martínez Sanz 147). Al igual que la creencia de que «los hombres no rechazan una oportunidad de relación sexual», tal y como dice Félix López Sánchez, «además de ser falsa, les animaliza y puede servirles de coartada para legitimar la coerción y la violencia sexual» (22). Por ello, tampoco los hombres perciben esta violencia, tal y como se puso de manifiesto en una investigación realizada con 229 hombres

Feminismo/s 31, junio 2018, pp. 127-141 
que estaban en un programa de intervención por malos tratos, en el que el 53\% habían asaltado sexualmente a sus mujeres, sin embargo, tan solo el 8\% lo percibía como un abuso sexual (Bergen y Bukovec).

Esta ausencia de percepción se ha visto corroborada por diferentes estudios. Algunas investigaciones han concluido que la relación de parentesco que existe con el perpetrador de la agresión sexual influye en la percepción de gravedad del asalto por parte de la víctima, siendo menor conforme crece la familiaridad con el agresor (Monson, Langhinrichsen- Rohling y Binderup). Por ejemplo, en la Macroencuenta de violencia contra la mujer 2015 (Delegación del Gobierno para la Violencia de Género), exponen que el motivo principal que aluden las mujeres para no interponer denuncia contra sus parejas es precisamente que no le concedían la suficiente importancia a la violencia vivida, es decir, no perciben la gravedad de la misma.

La violencia sexual sobre las mujeres en la pareja está tolerada y favorecida por el contexto sociocultural, incluido el ámbito del derecho que tampoco percibe esta violencia. En este sentido, Ramos-Lira et al. sostienen que «a veces las mujeres no reconocen la violencia sexual que han experimentado como una violación o como otro tipo de abuso debido a que el contexto sociocultural normaliza, minimiza, tolera o promueve estos actos» (183). Así mismo, añaden que, en ocasiones, las mujeres no lo cuentan por miedo a la reacción de los y las profesionales o por las consecuencias que pueda tener su denuncia. De hecho, la percepción social de que las mujeres tienen que asumir el débito conyugal llega a tal extremo que, en muchos países del mundo, los abusos sexuales y la violación cometida por el compañero íntimo no se considera un delito, sino que está asumido que, una vez firmado el contrato de matrimonio, el marido tiene derecho a exigir a su esposa relaciones sexuales ilimitadas (UNICEF). Podemos ver titulares en la prensa nacional e internacional sobre países de la Unión Europea, como Lituania, Estonia, Hungría y Bulgaria, entre otros que, pese a las recomendaciones de instituciones internacionales, no tipifican como delito el sexo forzado en el matrimonio (Sahuquillo). En los países en que está considerado como un delito, a las mujeres les resulta difícil presentar una acusación a causa del rígido régimen probatorio relacionado con el delito.

Algunas personas pueden llegar a pensar que las mujeres acceden a tener relaciones sexuales sin desearlo por condescendencia hacia sus parejas. Sin

Feminismo/s 31, junio 2018, pp. 127-141 
embargo, por lo argumentado hasta aquí, es evidente que se trata de violencia sexual y, como tal, es una clara forma de ejercer la violencia de género, fruto de la cultura machista y patriarcal que, tal y como defiende Galtun, cumple con los criterios para ser estructural, cultural y directa.

La falta de visibilidad de la violencia sexual es causa y consecuencia de la falta de investigación en este ámbito, de la falta de exploración por parte de los profesionales que pueden detectar esta forma de violencia y la falta de herramientas para poder explorarla (Martínez Sanz 345). Mahoney y su equipo, en su estudio de revisión de 2007, describen las barreras que existen para la evaluación de la violencia sexual, para el estudio de su prevalencia y para visibilizar a las víctimas, éstas son: la lealtad que las mujeres sienten hacia su pareja y el deber de mantener la privacidad, las dificultades para aceptar la propia victimización, la reticencia a utilizar la etiqueta de «violación», los malentendidos sobre el papel de las mujeres en las responsabilidades del matrimonio, inexperiencia sexual e incertidumbre para diferenciar cuando una relación sexual es «normal»o «forzada». Por ello, concluyen que no hay apenas estudios que incluyan en su diseño cuestiones para establecer la prevalencia de los asaltos sexuales dentro de la pareja, pero también son escasos los estudios sobre las características y las consecuencias de este tipo de violencia. Rachel Kennedy expone la necesidad de realizar estudios de ámbito nacional en distintas poblaciones de mujeres sobre violencia sexual en la pareja, porque las necesidades de estas mujeres deben ser mejor atendidas.

El estudio de revisión que realizaron Bagwell-Gray, Messing y BaldwinWhite en 2015 critica las siete herramientas más utilizadas en Estados Unidos para evaluar la violencia de género, por su incapacidad para medir la violencia sexual en la relación de pareja, bien por la baja fiabilidad, porque no especifica que la violencia sexual sea ejercida por la pareja, o porque no exploran todas las formas de violencia sexual dentro de la relación de pareja. Sin embargo, hay una herramienta, la Entrevista Semiestructurada para la Exploración de la Violencia Sexual en la Relación de Pareja (EVS) (Martínez Sanz, Mañas Viejo y Pons Salvador) ${ }^{5}$ que explora en profundidad

5. La EVS es una herramienta de recogida de información sobre la violencia sexual en la pareja, que tiene el objetivo de facilitar a las y los profesionales que trabajan como 
si las mujeres mantienen relaciones sexuales sin desearlo, qué tipo de conductas sexuales realizan, cuál es el método de coacción que utilizan las parejas, cómo reaccionan ellas en estas situaciones, así como explora otra información sobre su salud sexual.

\section{CONCLUSIONES}

De lo expuesto hasta aquí, es posible concluir que, aunque es muy difícil detectar la violencia sexual que se ejerce sobre las mujeres en la relación de la pareja por la ausencia reconocimiento social, son muchas las investigaciones que demuestran que es un problema muy grave que está experimentado un gran número de mujeres. Esta invisibilidad se ve favorecida y tolerada por las creencias asociadas a los roles de género, en concreto a los que de forma estereotipada se asignan a la sexualidad de las mujeres, que influyen y predisponen el comportamiento sexual, induciendo las situaciones de violencia sexual en la pareja. Entre estas creencias, cobra protagonismo el «débito conyugal» que obliga a las mujeres a satisfacer las necesidades sexuales de sus parejas, aunque sus propias necesidades y derechos queden totalmente vulnerados. Las mujeres perciben de manera más o menos inconsciente que transgreden su rol femenino si se niegan, se sienten culpables y acceden creyendo que es una obligación autoimpuesta. No importa, por tanto, si lo hacen con o sin consentimiento, por presiones o convicciones culturales, porque no son conscientes de los mecanismos y las consecuencias reales de esta violencia. Los hombres, por su parte, justifican su necesidad y exigen que sea satisfecha, lo que conlleva el que ejerzan presión o coacción sobre sus parejas.

Tal y como hemos visto, estas creencias proceden de la sociedad machista y patriarcal que justifica y promueve esta violencia. La gran ausencia de conocimientos específicos sobre la sexualidad femenina, el control y la coacción que se ejerce sobre ella, está basada fundamentalmente en estereotipos de roles y falsas creencias con profundas raíces judeo-cristianas. En este contexto, en el que lo femenino no es relevante, también a las y los profesionales les cuesta poner el foco en la sexualidad de las mujeres más allá de

mujeres que han vivido una situación de violencia de género la detección de esta forma de violencia. Está basada en una revisión exhaustiva de la literatura científica y profesional, así como la experiencia clínica.

Feminismo/s 31, junio 2018, pp. 127-141 
su función reproductora. Debido a estos motivos no la exploran, no existen apenas herramientas para explorarla y, consecuentemente, no se investiga lo suficiente, por lo que la violencia sexual dentro de la relación de pareja se hace invisible.

Por ello, las investigaciones que estudien en profundidad y aumenten la percepción de la violencia sexual en la relación de pareja adquieren todo su sentido. Al igual que ha pasado con la violencia física y psicológica en la pareja, que pasó de ser algo privado a un problema social y un delito, debemos esforzarnos para visibilizar, estimar y describir la violencia sexual sobre las mujeres en la relación de pareja, así como conocer la repercusión de ésta en la salud.

Debemos condenar, combatir y reducir todas las formas de violencia relacionadas con la sexualidad (WAS 18). Por ello, nos unimos a Marlene García cuando afirma que las y los profesionales debemos tener un papel activo en la reestructuración de los estereotipos que constituyen un sistema encubierto y silenciador que coloca a las mujeres en una situación de vulnerabilidad por el mero hecho de serlo. Tal y como dice esta autora, «si ellas que lo sienten no lo ven, debemos [las y] los profesionales esforzarnos en sensibilizarnos, conocerlos y visibilizarlos al máximo» (490).

\section{REFERENCIAS BIBLIOGRÁFICAS}

Asensio Cerver, Francisco. Tratado médico familiar de la sexualidad en la mujer. Anatomía, fisiología, patología. Barcelona: Guías Atrium de medicina y salud, 2000.

Bagwell-Gray, Meredith E., Jill Theresa Messing y Adrienne Baldwin-White. «Intimate partner sexual violence: A review of terms, definitions, and prevalence». Trauma Violence \& Abuse 16.3 (2015): 316-335. doi: $10.1177 / 1524838014557290$

Basile, Kathleen C. «Prevalence of wife rape and other intimate partner sexual coercion in a nationally representative sample of women». Violence and Victims 17.5 (2002): 511-524. doi: http://dx.doi.org/10.1891/vivi.17.5.511.33717

Bergen, Raquel Kennedy y Paul Bukovec. «Men and intimate partner rape: characteristics of men who sexually abuse their partner». Journal of Interpersonal Violence 21.10 (2006): 1375-1384. doi: 10.1177/0886260506291652 
Cano Martín, Luis Miguel, Alberto Berrocoso Martínez, Leonor de Arriba Muñoz, Rodrigo Bernaldo de Quirós Lorenzana, Juan Diego Alamar Provecho y Ana Cardo Maza. «Prevalencia de violencia de género en mujeres que acuden a consultas de atención primaria». Revista Clínica de Medicina de Familia 3.1 (2010): 10-17. http://scielo.isciii.es/pdf/albacete/v3n1/original2.pdf

Coker, Ann L., Paige Hall Smith, Robert E. McKeown y Melisa J. King. «Frequency and correlates of intimate partner violence by type: physical, sexual, and psychological battering». American Journal of Public Health 90.4 (2000): 553-559. http://www.ncbi.nlm.nih.gov/pmc/articles/PMC1446198/pdf/10754969.pdf

Delegación del Gobierno para la Violencia de Género. Sobre la Inhibición a Denunciar de las Víctimas de Violencia de Género. Madrid: Ministerio de Sanidad, Servicios Sociales e Igualdad, 2015. http://www.msssi.gob.es/ssi/ violenciaGenero/publicaciones/estudiosinvestigaciones/PDFS/Inhibicion_ Denunciar_VictimasVG.pdf

Durán, M. Mercedes. Sexismo benévolo y violencia sexual: percepción social de la violación en relaciones intimas. Tesis Doctoral, Universidad de Granada, 2010. http://hera.ugr.es/tesisugr/18695620.pdf

Fontanil, Yolanda, Esteban Ezama, Roxana Fernández, Pura Gil, Francisco Javier Herrero y Dolores Paz. «Prevalencia del maltrato de pareja contra las mujeres». Psicothema 17.1 (2005): 90-95. http://www.psicothema.com/pdf/3069. pdf

Galtung, Johan. Investigaciones teóricas. Sociedad y cultura contemporáneas. Madrid: Instituto de Cultura «Juan Gil-Albert», 1995. 314-328.

García Benavides, Marlene. «Aspectos psicológicos sobre la violencia de género». Manual de lucha contra la violencia de género. Ed. Antonio Nicolás Marchal Escalona. Navarra: Editorial Aranzadi, 2010. 479-328.

Instituto de la Mujer. Delitos conocidos contra la libertad e indemnidad sexual cometidos en el ámbito familiar, según relación víctima-agresor. Delitos contra la libertad sexual. Ministerio de Trabajo y Asuntos Sociales, 2009. www.inmujer.es

Instituto Nacional de las Mujeres. Violencia sexual contra las mujeres en el seno de la pareja conviviente. México, 2006. http://cedoc.inmujeres.gob.mx/documentos_download/100790.pdf

Jewkes, Rachel, Purna Sen y Claudia García-Moreno. «Sexual violence». World report on violence and health. Eds. Etienne G. Krug, Linda L. Dahlberg, James A. Mercy, Anthony B. Zwi \& Rafael Lozano. Ginebra: Organización Mundial de la Salud, 2002. 147-181. http://apps.who.int/iris/ 
bitstream/handle/10665/42495/9241545615_eng.pdf;jsessionid= 81377ED9CA313AFE45DB95F8047D6A21? sequence $=1$

Kennedy Bergen, Rachel. «Studying wife rape: reflections on the past, present, and future». Violence Against Woman 10.12 (2004): 1407-1416.

Labrador, Francisco Javier, Paulina Paz Rincón, Pilar de Luis y Rocío FernándezVelasco. Mujeres Victimas de la violencia doméstica. Programa de actuación. Madrid: Psicología Pirámide, 2004/2008.

López Mondéjar, Lola. «Pegarse/ des-pegarse. El vínculo amoroso de la violencia de género». Violencia y agresividad. Fundamento para la prevención psicosocial. Eds. Francisco J. Coll y E. Fernández. Mancomunidad de Municipios del Valle de Ricote. Centro de estudios e investigación psicosocial, 2003. 119-135.

López Sánchez, Félix. Ética de las relaciones sexuales y amorosas. Madrid: Psicología Pirámide, 2015.

Mahoney, Patricia y Linda M. Williams. «Sexual assault in marriage: Prevalence, consequences, and treatment of wife rape». Partner Violence: A 20-year Literature Review and Synthesis (2007): 1-43. http://brockbaker.pbworks. com/f/PartnerViolence.pdf

Martínez Sanz, Alicia (2015). Estudio de la violencia sexual sobre las mujeres en la relación de pareja, y las repercusiones de la violencia en la pareja sobre la sexualidad de las mujeres. Estudio realizado en servicios públicos de la Comunidad Valenciana. Tesis Doctoral, Universidad de Alicante, 2015. https://rua.ua.es/ dspace/bitstream/10045/64881/1/tesis_martinez_sanz.pdf

Martínez Sanz, Alicia, Carmen Mañas Viejo y Nieves Montesinos Sánchez. «Maltrato sexual sobre las mujeres en sus relaciones de pareja: un estudio de caso». Asparkía 24 (2013): 88-103. http://www.e-revistes.uji.es/index.php/ asparkia/article/view/1164/1040

Martínez Sanz, Alicia, Carmen Mañas Viejo y Gemma Pons Salvador. «Entrevista Semiestructurada para la Exploración de la Violencia Sexual en la relación de pareja (EVS)». Información Psicológica 111 (2016): 18-34. https://rua.ua.es/ dspace/bitstream/10045/57473/1/2016_Martinez-Sanz_etal_InfPsicol.pdf

Monson, Candide M., Jennifer Langhinrichsen-Rohling y Tisha Binderup. «Does 'no' really mean 'no' after you say 'yes'?». Journal of Interpersonal Violence 15.11 (2000): 1156-1174. doi: 10.1177/088626000015011003

Organización Mundial de la Salud (OMS). Estudio multipaís de la OMS sobre la saludy la violencia doméstica: primeros resultados sobre prevalencia, eventos relativos a la 
salud y respuestas de las mujeres a dicha violencia: Resumen del informe. Ginebra, 2005. http://apps.who.int/iris/bitstream/10665/43390/1/924359351X_spa.pdf Organización Mundial de la Salud (OMS). Defining sexual health: report of a technical consultation on sexual health, 28-31 January 2002, Geneva, 2006. http://www.who.int/reproductivehealth/publications/sexual_health/defining_ sexual_health.pdf

Organización Panamericana de la Salud (OPS). Informe mundial sobre la violencia y la salud. Resumen. Washington, D. C.: Oficina Regional para las Américas de la Organización Mundial de la Salud, 2002. http://www.who. int/violence_injury_prevention/violence/world_report/es/summary_es.pdf

Ramos-Lira, Luciana, María Teresa Saltijeral-Méndez, Martha Romero-Mendoza, Miguel Ángel Caballero-Gutiérrez y Nora Angélica Martínez-Vélez. «Violencia sexual y problemas asociados en una muestra de usuarias de un centro de salud». Salud Pública de México 43.3 (2001): 182-191. http://www.scielo.org. $\mathrm{mx} / \mathrm{pdf} / \mathrm{spm} / \mathrm{v} 43 \mathrm{n} 3 / \mathrm{a} 02 \mathrm{v} 43 \mathrm{n} 3 . \mathrm{pdf}$

Sahuquillo, María R. «Violación conyugal sin condena». El País 8 de marzo de 2015. https://elpais.com/internacional/2015/03/08/actualidad/1425845224_956206.html

Saltijeral, María Teresa, Luciana Ramos y Miguel Ángel Caballero. «Las mujeres que han sido víctimas de maltrato conyugal: Tipos de violencia experimentada y algunos efectos en la salud mental». Salud Mental 21.2 (1998): 10-18. http://www.revistasaludmental.mx/index.php/salud_mental/article/ view/697/696

Sanmartin Esplugues, José, Isabel Iborra Marmolejo, Yolanda García Esteve y Pilar Martínez Sánchez. III informe internacional. Violencia contra la mujer en las relaciones de pareja. Estadística y legislación. Valencia: Centro Reina Sofía. Valencian International University, 2010.

Santos Iglesias, Pablo y Juan Carlos Sierra. «El papel de la asertividad sexual en la sexualidad humana: Una revisión sistemática». International Journal of Clinical and Health Psychology 10.3 (2010): 553-577. http://www.aepc.es/ ijchp/articulos_pdf/ijchp-366.pdf

UNICEF. La violencia doméstica contra mujeres y niñas. Fondo de Naciones Unidas para la Infancia. Florencia: Centro de Investigaciones Innocenti, 2000. http:// www.unicef-irc.org/publications/pdf/digest6s.pdf 
World Association of Sexology (WAS). Declaración de los derechos sexuales. Declaración del $13^{\circ}$ Congreso mundial de sexología. Valencia, 1997. Revisada y aprobada por la Asamblea General de la WAS, el 26 de agosto de 1999, en el $14^{\circ}$ Congreso mundial de sexología, Hong Kong, República Popular China. http://www.worldsexology.org/sites/default/files/Declaration\%20 of\%20Sexual\%20Rights.pdf

Feminismo/s 31, junio 2018, pp. 127-141 